\title{
ENGEVISTA
}

Página da revista: http://www.uff.br/engevista/seer/

\section{Avaliação do crescimento da microalga Scenedesmus sp.em diferentes concentrações de $\mathrm{NaCl}$ Microalgae growth rate Scenedesmus sp. in different $\mathrm{NaCl}$ concentration}

\author{
Maniza Sofia Monteiro Fernandes ${ }^{1}$ \\ Kepler Borges França ${ }^{2}$ \\ Rodrigo Vieira Alves ${ }^{3}$ \\ Howard William Pearson ${ }^{4}$ \\ Sonáli Amaral Lima ${ }^{5}$ \\ Tereziana Silva Costa ${ }^{6}$ \\ Bruna Da Silveira ${ }^{7}$
}

\begin{abstract}
Resumo: A junção de fatores biológicos, físicos e químicos influencia na obtenção de biomassa e de produtos metabólicos das microalgas. No âmbito dos fatores biológicos podem-se destacar as taxas metabólicas características da espécie cultivada e a influência de outros microrganismos no crescimento microalgal. Já com relação aos aspectos físico-químicos, a iluminação, temperatura, salinidade e disponibilidade de nutrientes no meio irão influenciar na produção da microalga. Ao se desenvolver um meio de cultura é importante a observação dos seguintes fatores: concentração total de sal, fonte de carbono, fonte de nitrogênio, entre outros. Este trabalho foi desenvolvido com o objetivo de avaliar o crescimento de microalgas Scenedesmus em diferentes concentrações de sais. $\mathrm{O}$ cultivo das microalgas em diferentes concentrações de $\mathrm{NaCl}$ teve como intenção investigar quais as melhores faixas de sais que as microalgas conseguem se adaptar, estudando a influência no crescimento celular, velocidade específica de crescimento e o tempo de duplicação de cada experimento como
\end{abstract}

\footnotetext{
${ }^{1}$ UFCG - Universidade Federal de Campina Grande

${ }^{2}$ UFCG - Universidade Federal de Campina Grande

${ }^{3}$ UFCG - Universidade Federal de Campina Grande

${ }^{4}$ UFCG - Universidade Federal de Campina Grande

${ }^{5}$ UNINASSAU - Faculdade Maurício de Nassau

${ }^{6}$ UFCG - Universidade Federal de Campina Grande

${ }^{7}$ UFCG - Universidade Federal de Campina Grande
} 
ISSN: $1415-7314$

ISSN online: $2317-6717$

também a taxa de inibição de crescimento. Como resultados obteve que o percentual de inibição em concentrações mais baixas foi de $18,4 \%$ que é correspondente a $1 \mathrm{~g}$ de $\mathrm{NaCl}$ e o mais alto foi de $42,9 \%$ o de $5 \mathrm{~g}$ de $\mathrm{NaCl}$. A inibição do crescimento no Scenedesmus só ocorre quando o meio é estimulado com concentrações mais altas de sais.

Palavras-chave: água salobra, microalgas, crescimento celular.

\begin{abstract}
The combination of biological, physical and chemical factors influences the obtainment of biomass and metabolic products of microalgae. Under the biological factors, metabolic rates characteristics of cultivated species and the influence of other microorganisms in microalgal growth can be highlighted. In relation to the physico-chemical, lighting, temperature, salinity and nutrient availability in the middle will influence the production of microalgae. When developing a culture medium, it is important to note the following factors: total salt concentration, carbon source, nitrogen source, among others. This work has been developed to evaluate the growth of microalgae Scenedesmus in different salt concentrations. The cultivation of microalgae in different concentrations of $\mathrm{NaCl}$ was intended to investigate what the best salts of tracks that microalgae can adapt to study the influence on cell growth, the specific growth rate and doubling time of each experiment, as well as the growth inhibition rate. As a result, it was found that the percentage of inhibition at lower concentrations was $18.4 \%$; which is equivalent to $1 \mathrm{~g} \mathrm{NaCl}$; and the highest was $42.9 \%$ in the $5 \mathrm{~g} \mathrm{NaCl}$. Growth inhibition in Scenedesmus only occurs when the medium is stimulated at higher concentrations of salts.
\end{abstract}

Keywords: Brackish water, microalgae, cellular growth. 


\section{Introdução}

De acordo com Dias (2010), levando-se em conta que 95\% da água contida no planeta é proveniente dos oceanos, uma forma que é empregada para a obtenção de água potável é a dessalinização da água oriunda dos mares e águas subterrâneas. É observada na composição da água salina a presença dos íons de sódio $\left(\mathrm{Na}^{+}\right)$, magnésio $\left(\mathrm{Mg}^{2+}\right)$, sulfato $\left(\mathrm{SO}_{4}{ }^{2-}\right)$, bicarbonato $\left(\mathrm{HCO}_{3}{ }^{-}\right)$, cálcio $\left(\mathrm{Ca}^{2+}\right)$, potássio $\left(\mathrm{K}^{+}\right)$, cloreto $\left(\mathrm{Cl}^{-}\right)$, entre outros. Schmitz, et al., 2012 destacam que as microalgas são um grupo bastante diversificado de microrganismos. Tais seres são aquáticos, sendo na maioria das vezes microscópicos e unicelulares, possuem a capacidade de formação de colônia, não acontecendo, porém com todos os tipos de microalgas e apresentam pouca ou nenhuma diferenciação celular. Segundo relata Baumgartner et al. (2013) a palavra alga é utilizada para nomear uma grande variedade de microrganismos, os quais diferem em morfologia, reprodução, fisiologia e ecologia. Podem englobar seres unicelulares e multicelulares, sendo a maioria das algas em escala microscópica (microalgas) de hábitos planctônicos. A biomassa produzida no cultivo das microalgas pode ser utilizada em várias aplicações, podendo-se exemplificar a alimentação humana e animal com os suplementos alimentares, na indústria de cosméticos, no tratamento de águas residuárias e na produção de bicombustíveis. Tem sido visto ao longo dos últimos anos um grande crescimento nos processos e aplicações que envolvem o uso das microalgas, tendo como principal motivo as mais variadas substâncias sintetizadas por tais microrganismos, as quais possuem um grande valor no mercado mundial.

Mais especificamente sobre a microalga Scenedesmussp., Oliveira (2013) ressalta que são as microalgas mais encontradas no plâncton de águas doces e menos frequentemente em águas salobras. São seres clorofilados, unicelulares e uninucleadas, pertencentes à família Scenedesmaceae, gênero Scenedesmussp. Com relação à forma são elipsoidais, colônias planas e suas células se agrupam em número de 4, 8, ou em casos mais raros 16 ou 32 células. O teor de lipídeos da microalga Scenedesmussp. varia de 11 a $20 \%$, quando cultivada em condições padrões, e pode chegar a $70 \%$ quando o meio de cultivo é otimizado.

A junção de fatores biológicos, físicos e químicos influencia na obtenção de biomassa e de produtos metabólicos das microalgas. No âmbito dos fatores biológicos podem-se destacar as taxas metabólicas características da espécie cultivada e a influência de outros microrganismos no crescimento microalgal. Já com relação aos físico-químicos, a iluminação, temperatura, salinidade e disponibilidade de nutrientes no meio irão influenciar na produção da microalga.

Ainda sobre os fatores que influenciam na produção da microalga, Oliveira (2013) mostra que a intensidade luminosa, temperatura, $\mathrm{pH}$, aeração, concentração de nutrientes e concentração de $\mathrm{CO}_{2}$ causam impacto na composição da biomassa microalgal. No controle da variável temperatura, é desejável que a mesma permaneça constante, já que promove uma estabilidade maior nos experimentos e nos processos rotineiros. A faixa ótima de operação para as cepas é de 18 a $22^{\circ} \mathrm{C}$ e quando se deseja acelerar o crescimento celular das microalgas recomenda-se que se opere em 
temperaturas mais altas, dependendo do limite de tolerância da espécie. No quesito iluminação, a intensidade da luz, o comprimento de onda e a duração da iluminação às quais as células estarão submetidas fornecem uma condição ótima para o crescimento das microalgas. Geralmente se usam lâmpadas fluorescentes do tipo luz do dia, pois as mesmas simulam comprimentos de onda entre 350 e $700 \mathrm{~nm}$, que são necessários para a realização da fotossíntese. As reações que ocorrem em tal processo estão esquematizadas na Figura 1 que mostra as duas etapas: fotoquímica (dependente de luz) e química (independente de luz), etapa essa que depende diretamente dos produtos formados na etapa fotoquímica.

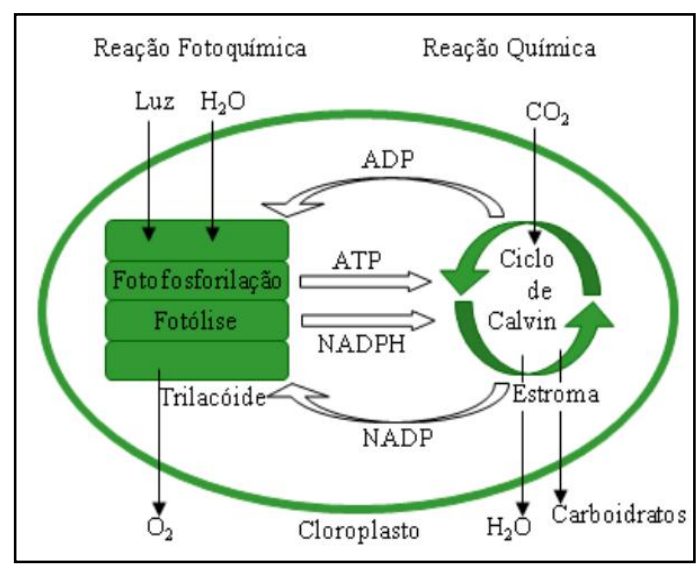

Figura 1. Reações da fotossíntese

Com relação à aeração, tal parâmetro está diretamente relacionado à demanda de carbono, já que quando se prepara os meios de cultivo não se adiciona a fonte de carbono. Outra utilidade da aeração é a agitação dos cultivos, o que vai permitir a homogeneização do meio, não permitindo a decantação e acumulação de células no fundo do recipiente.

A relevância do pH no processo é mostrada por Oliveira (2013), ressaltando que dependendo do valor de tal parâmetro pode aumentar ou diminuir a disponibilidade de vários elementos químicos, os quais podem cristalizar e precipitar no meio. Por isso a faixa de $\mathrm{pH}$ em que deve-se operar é neutra. Desse modo os componentes do meio irão ser efetivamente absorvidos pelas microalgas. Sabendo que na medida em que aumenta o crescimento da microalga aumenta também o consumo de $\mathrm{CO}_{2}$ dissolvido no meio isso irá acarretar em um aumento do $\mathrm{pH}$, ficando em um valor maior que 10. Portanto deve-se ter um controle rígido da disponibilidade de $\mathrm{CO}_{2}$, já que elevadas concentrações deste componente podem diminuir significativamente o $\mathrm{pH}$ do meio, inibindo assim o crescimento das células.

Quanto às condições nutricionais, Santos (2011) ressalta que as duas principais formas de nutrição que as microalgas possuem são a autotrófica e heterotrófica, sendo a autotrófica mais importante. Em tal forma de nutrição a energia química necessária é obtida através da fotossíntese, processo em que há conversão da energia solar em energia química. Isso acarreta a redução do $\mathrm{CO}_{2}$ para a glicose ser sintetizada através da oxidação de substratos, principalmente água, liberando, assim $\mathrm{O}_{2}$. É importante ainda observar a grande capacidade de absorção dos nutrientes em concentrações 
ISSN: 1415-7314

ISSN online: $2317-6717$

bem pequenas que as microalgas possuem. Também se observa que elementos que estão presentes abundantemente no meio extracelular são mais facilmente acumulados dentro da célula. Sendo assim, a composição intracelular das microalgas reflete o meio de cultura aos quais as mesmas estão sendo submetidas, bem como a disponibilidade de tais elementos nutrientes. Logo, as proporções dos nutrientes elementares da célula estão sujeitos à variações, a depender da disponibilidade dos nutrientes do meio de cultura.

Para o crescimento das microalgas em taxas satisfatórias deve ser observado ao condicionamento do inoculo, para que se atenda ao maior número possível de exigências que o microrganismo possui e forneça os nutrientes necessários para o crescimento e síntese celular. Ao se desenvolver um meio de cultura é importante a observação dos seguintes fatores: concentração total de sal, fonte de carbono, fonte de nitrogênio, entre outros. Falando dos meios de cultura sintéticos, o elevado custo dos reagentes utilizados na preparação destes se mostra um problema a ser solucionado ou minimizado no cultivo das microalgas. Desse modo, o fator econômico deve ser aliado à demanda dos nutrientes que os microrganismos necessitam para um crescimento satisfatório (BAUMGARTNER et al., 2013).

Baumgartner et al.(2013) mostraram teste de crescimento da microalga Scenedesmusacuminatus nos meios de cultivo: MC (Medium for Chlorellaellipsoidea), DM (Detmer'sMedium) eNPK (Nitrogen:Phosphorus:Potassium) nas proporções mássicas 10:10:10 e 20:5:10. Como resultado do estudo o meio de cultivo que apresentou maior produtividade da biomassa foi o meio MC e a taxa de crescimento dos meios NPK (10:10:10) e NPK (20:05:10) se mostraram menores que os meio DM e MC.

Variadas espécies de microalgas podem ser utilizadas para produção em grande escala de biodiesel, todavia, Cunha et al. (2009) e Lourenço et al. (2010) explicam que fatores como a velocidade de crescimento e da sua composição química devem ser levados em consideração na escolha de cepas mais adequadas, uma vez que são influenciados pelo meio de cultura utilizado, a idade do cultivo, a intensidade luminosa, a temperatura, a salinidade e o fotoperíodo.

Este trabalho foi desenvolvido com o objetivo de avaliar o crescimento de microalgas Scenedesmussp.em diferentes concentrações de sais.

\section{Material e métodos}

A cepa utilizada foi Scenedesmussp. proveniente do laboratório de Ambientes Recifais e Biotecnologia com Microalgas (LARBIM) da Universidade Federal da Paraíba, mantida no cepário do Laboratório de Referência em Dessalinização (LABDES)da Universidade Federal de Campina Grande, onde foram realizados os testes. Para o cultivo das microalgas foi utilizado o meio de cultura WC, aeração constante, a fim de manter os cultivos homogeneizados, iluminação artificial contínua através de lâmpadas fluorescentes de $40 \mathrm{~W}$ e temperatura controlada de $25^{\circ} \mathrm{C}$. 
Os testes foram realizados em triplicata variando-se as concentrações de cloreto de sódio $(\mathrm{NaCl})$ entre 1 e $5 \mathrm{~g} / \mathrm{L}$, com intuito de viabilizar a adaptação das microalgas em diferentes proporções de sais. O crescimento algáceo foi determinado a partir de contagens com câmara de Neubauer em microscópio óptico e, também foram determinados a velocidade específica de crescimento e o tempo de duplicação na fase exponencial de crescimento (fase log).

Para verificar a existência de diferenças estatisticamente significativas entre o controle no meio Water Culture (WC)e as amostras(suplementação de $\mathrm{NaCl}$ ) foi aplicada a Análise de Variância (ANOVA) seguida de Teste de Tuckey, com intervalode confiança de $95 \%(\alpha=0,05)$. Para a análise estatística, utilizou-se o software MNITAB ${ }^{\circledR} 16.0$.

\section{Resultados e discussão}

O cultivo das microalgas em diferentes concentrações de $\mathrm{NaCl}$ teve como intenção investigar quais as melhores faixas de sais que as microalgas conseguem se adaptar, estudando a influência no crescimento celular, velocidade específica de crescimento e o tempo de duplicação de cada experimento como também a taxa de inibição de crescimento.

$\mathrm{Na}$ fase lag é comum ocorrer algum tipo de inibição ao iniciar o crescimento, uma vez que a cultura recém-inoculada se adapta ao novo ambiente. Nos experimentos realizados ocorreram pouca ou nenhuma divisão celular nesta fase, pois as células se encontravam em estado de latência, decorrente da mudança das condições de cultivo. Os experimentos iniciaram com uma concentração inicial da ordem de $10^{5}$ cel./mL e até atingir a fase estacionária percebeu-se um crescimento satisfatório pela mudança da cor do inoculo devido ao crescimento exponencial das células, conforme demonstra a Figura 2.

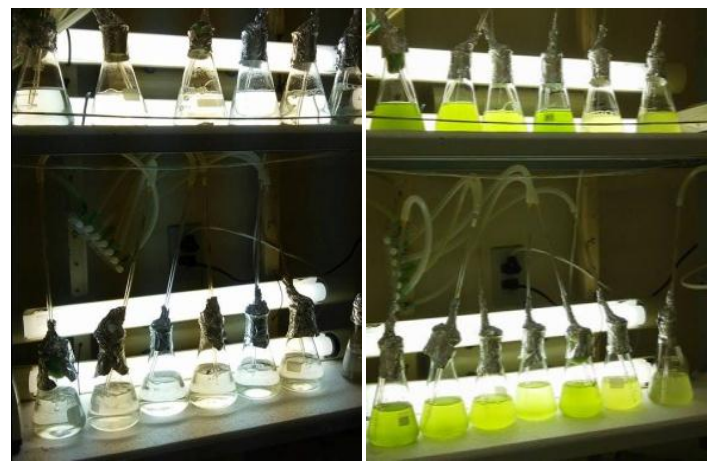

Figura 2. Mudança de cor dos experimentos devido ao crescimento das microalgas

Analisando a Figura 3 observa-se o crescimento celular da Scenedesmussp. Cultivada no meio WC, sendo que a representação das curvas se refere à densidade celular média diária, na qual a fase de adaptação começou com uma população da ordem de $10^{5}$ cel. $\mathrm{mL}^{-1}$, até chegar a uma fase estacionaria de $10^{7} \mathrm{cel} . \mathrm{mL}^{-1}$. 

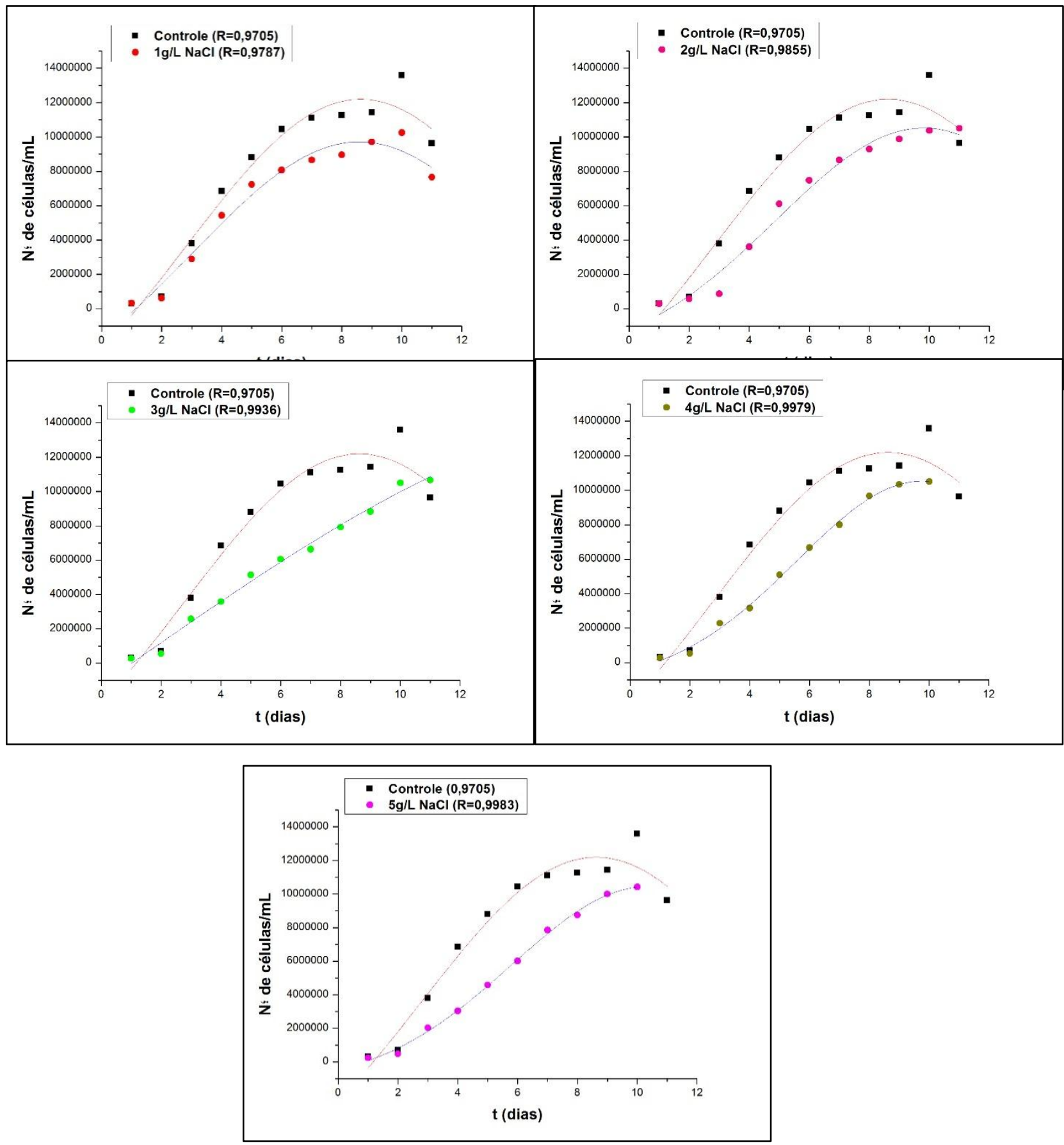

Figura 3. Curvas de crescimento das microalgas com variação de $\mathrm{NaCl}$.

A Análise de Variância (ANOVA) para o crescimento celular foi realizada utilizando-se o número de células no $9^{\circ}$ dia de experimento, tendo em vista que o controle já havia alcançado a fase estacionária. Para este parâmetro foi verificada diferença estatisticamente significativa $(p=0,029)$ com 95\% de confiança, assim como para a velocidade específica de crescimento $(\mathrm{p}=0,000)$ e o tempo de duplicação $(\mathrm{p}=0,000)$ mostrados na Tabela 1 .

Além das concentrações celulares médias no $9^{\circ}$ dia de cultivo, velocidade específica de crescimento e tempo de duplicação determinados na fase exponencial, a Tabela 1 apresenta o 
resultado do teste de Tuckey, onde letras diferentes na mesma coluna representam médias estatisticamente diferentes.

O teste de Tuckey mostra que nenhum dos cultivos com adições de sais foi estatisticamente semelhante ao controle, além disso, o cultivo com adição de $3 \mathrm{~g}$ de $\mathrm{NaCl}$ também apresentou diferença com relação aos demais experimentos para a concentração celular e o tempo de duplicação.

De forma geral todos os experimentos apresentaram menor concentração celular, menor velocidade específica de crescimento e consequentemente maior tempo de duplicação com relação ao controle, no entanto, observa-se que com o aumento da concentração de sais os resultados desses parâmetros tendem a se aproximar dos resultados obtidos para o controle, o que demonstra a possibilidade de adaptação da Scenedesmussp. em águas com certas concentrações de sais.

A adaptação dessas microalgas, assim como o estudo de sua composição tem grande importância tecnológica. De acordo com os resultados obtidos por Salamaet al. (2013), a biomassa seca e o teor de lipídeos dessas microalgas foram melhorados quando cultivadas em condições salinas (0 a $25 \mathrm{mM}$ de $\mathrm{NaCl}$ ), o que pode representar uma otimização do cultivo para produção de biocombustíveis.

Tabela 1. Resultados com aplicação do teste de Tuckey

\begin{tabular}{cccc}
\hline & Concentração(cél./mL) & $\boldsymbol{\mu ( \mathbf { 1 } / \mathbf { h } )}$ & td (h) \\
\hline Controle & $1,14 \mathrm{E}+07^{\mathrm{A}}$ & $0,0232^{\mathrm{A}}$ & $30,09^{\mathrm{C}}$ \\
$\mathbf{1} \mathbf{g}$ & $9,71 \mathrm{E}+06^{\mathrm{AB}}$ & $0,0146^{\mathrm{B}}$ & $47,37^{\mathrm{A}}$ \\
$\mathbf{2} \mathbf{g}$ & $9,88 \mathrm{E}+06^{\mathrm{AB}}$ & $0,0147^{\mathrm{B}}$ & $47,17^{\mathrm{A}}$ \\
$\mathbf{3} \mathbf{g}$ & $8,83 \mathrm{E}+06^{\mathrm{B}}$ & $0,0154^{\mathrm{B}}$ & $45,15^{\mathrm{AB}}$ \\
$\mathbf{4} \mathbf{g}$ & $1,03 \mathrm{E}+07^{\mathrm{AB}}$ & $0,0176^{\mathrm{B}}$ & $39,31^{\mathrm{B}}$ \\
$\mathbf{5} \mathbf{g}$ & $1,00 \mathrm{E}+07^{\mathrm{AB}}$ & $0,0183^{\mathrm{B}}$ & $38,30^{\mathrm{B}}$ \\
\hline
\end{tabular}

Através dos dados da densidade celular inicial e final foi feita o gráfico para comparar a inibição de crescimento de microalgas (Figura 4) nas diferentes adições de $\mathrm{NaCl}$. A concentração mais baixa foi de $18,4 \%$ que correspondente a $1 \mathrm{~g}$ de $\mathrm{NaCl}$ e o mais alto foi de $42,9 \%$ o que corresponde a uma adição de $5 \mathrm{~g}$. A inibição do crescimento no Scenedesmus só ocorre quando o meio é estimulado com concentrações mais altas de sais. Observou-se que a suplementação de $\mathrm{NaCl}$ não inibiu o crescimento das microalgas, porém houve um atraso de crescimento nas amostras em relação ao controle. Esses resultados foram semelhantes aos obtidos por Ferreira (2012), a qual utilizou o concentrado da separação por membrana de águas salobras para o cultivo de Clorellas $s p$. 


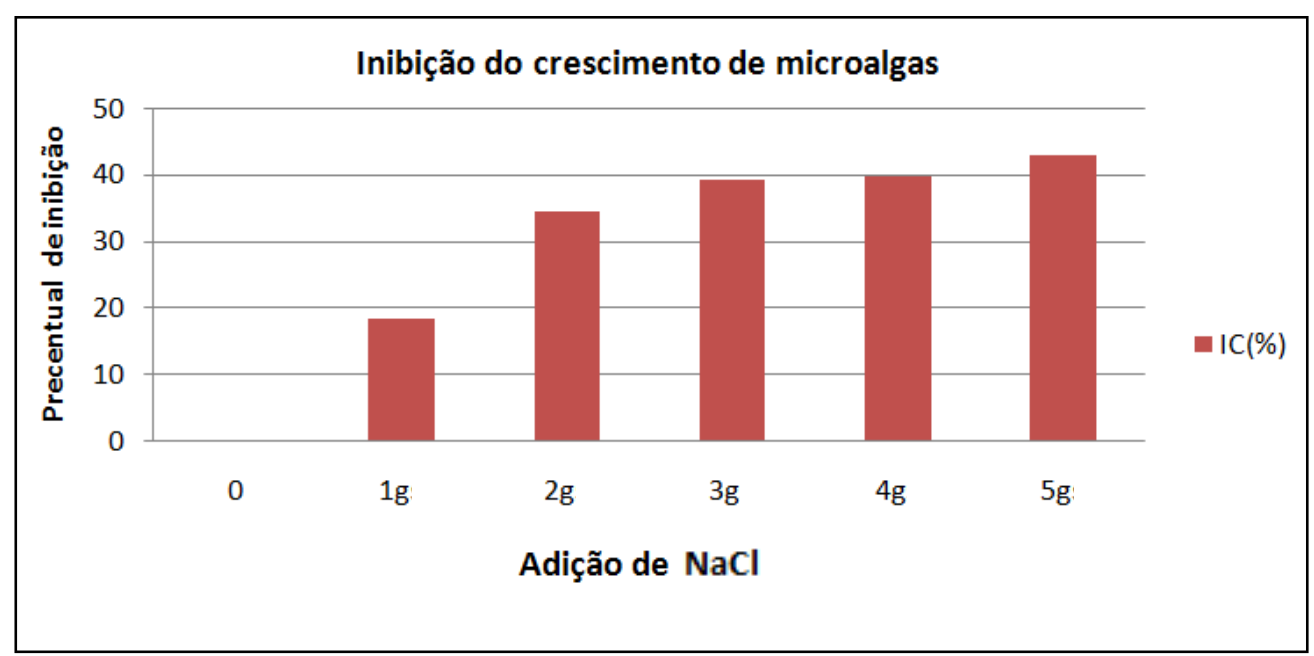

Figura 4. Inibição de crescimento de microalgas

\section{Conclusões}

A suplementação de $\mathrm{NaCl}$ não inibiu o crescimento das microalgas, porém houve um atraso de crescimento nas amostras em relação ao controle. Houve um atraso no crescimento das microalgas nos meios suplementados de $\mathrm{NaCl}$ com relação ao controle, no entanto, observa-se que com o aumento da concentração de sais os resultados desses parâmetros tendem a se aproximar dos resultados obtidos para o controle, o que demonstra a possibilidade de adaptação da Scenedesmussp.em águas salobras, possibilitando o uso dessa técnica para tratamento de águas com concentrações altas de sais.

\section{Referências}

CUNHA, D. C.; CREXI, V. T.; PINTO, L. A. A. Sinterização de óleo de pescado via solvente. Ciência e Tecnologia de Alimentos, Campinas, v.29, n. 1, pp. 207-213, Jan. 2009.

DIAS, J. R. Modelo de transformação de energia eólica num fluxo de água com alta pressão para dessalinização por Osmose Reversa ou/e geração de eletricidade. 2010. 221f. Tese (Doutorado em Engenharia) - Escola Politécnica da Universidade de São Paulo Escola Politécnica, Universidade de São Paulo, São Paulo. 2010.

LOURENÇO, S. O.; CAMPOS, V. B.; BARABARINO, E. Crescimento e composição química de dez espécies de microalgas marinhas em cultivos estanques. Ciência Rural, Santa Maria, v. 40, n. 2, p. 339-347, Fev, 2010.

RADMANN, E. M.; Costa, J. A. V. Conteúdo lipídico e composição de ácidos graxos de microalgas expostas aos gasesCO2, SO2 e NO. Química Nova, v. 31 (7), p. 1609:1612, 2008.

SALAMA, E.; Kim, H.; Abou-Shanab, R. A. I.; Ji, M.; Oh, Y.; Kim, S.; Jeon, B. Biomass, lipid content, and fatty acid composition of fresh water Chlamy domonas mexicana and cenedesmusobliquus grown under salt stress. Bioprocess and Biosystems Engineering.v. 36, $\mathrm{n}^{\circ}$. 6, p. 827-833. Jun. 2013.http://link.springer.com/article/10.1007/s00449-013-0919-1

SCHMITZ, R.; Magro, C.D.; Colla, L. M. Aplicações Ambientais de Microalgas. CIATEC, vol.4(1), p.48-60,2012. 
ISSN: $1415-7314$

ISSN online: $2317-6717$ 